\title{
A machine-learning classifier trained with microRNA ratios to distinguish melanomas from nevi
}

Rodrigo Torres ${ }^{1}$, Ursula E Lang ${ }^{1,2}$, Miroslav Hejna ${ }^{5,6}$, Samuel J Shelton ${ }^{3}$, Nancy M Joseph ${ }^{2}$, A. Hunter Shain $^{1}$, Iwei Yeh ${ }^{1,2}$, Maria L. Wei ${ }^{1,4}$, Michael C Oldham ${ }^{3}$, Boris C Bastian ${ }^{1,2}$, Robert L Judson-Torres ${ }^{1 *}$

${ }^{1}$ Department of Dermatology, ${ }^{2}$ Department of Pathology, ${ }^{3}$ Department of Neurological Surgery University of California, San Francisco, CA 94143, ${ }^{4}$ San Francisco Veterans Affairs Medical Center, 5 Department of Physics, ${ }^{6}$ Carl R. Woese Institute for Genomic Biology, University of Illinois at UrbanaChampaign, Urbana, IL, 61801

Running Title: Feature selection for melanoma microRNA profiling

Financial Support: This research was funded in part by NIH DP5OD019787, the Sandler Foundation Program for Breakthrough Biomedical Research Fellowship, the UCSF Marcus Program in Precision Medicine Innovation Fund and the UCSF Helen Diller Impact Award.

*Corresponding Author

Robert L Judson-Torres

Department of Dermatology

Helen Diller Family Comprehensive Cancer Center

University of California San Francisco

San Francisco CA USA 94158

Tel. No. 415-476-7290

Email: judsontorreslab@gmail.com

Conflicts of interest

The authors declare no potential conflicts of interest. 


\begin{abstract}
The use of microRNAs as biomarkers has been proposed for many diseases including the diagnosis of melanoma. Although hundreds of microRNAs have been identified as differentially expressed in melanomas as compared to benign melanocytic lesions, limited consensus has been achieved across studies, constraining the effective use of these potentially useful markers. In this study we quantified microRNAs by next-generation sequencing from melanomas and their adjacent benign precursor nevi. We applied a machine learning-based pipeline to identify a microRNA signature that separated melanomas from nevi and was unaffected by confounding variables, such as patient age and tumor cell content. By employing the ratios of microRNAs that were either enriched or depleted in melanoma compared to nevi as a normalization strategy, the classifier performed similarly across multiple published microRNA datasets, obtained by microarray, small RNA sequencing, or RT-qPCR. Validation on separate cohorts of melanomas and nevi correctly classified lesions with $83 \%$ sensitivity and $71-83 \%$ specificity, independent of variation in tumor cell content of the sample or patient age.
\end{abstract}

Keywords: microRNA, melanoma, feature selection, molecular pathology 


\section{Introduction}

Misdiagnosis of cutaneous melanoma is among the most significant contributors to medical malpractice lawsuits in the United States (Wallace et al. 2013). The advanced stages of melanoma are associated with five-year survival rates less than $20 \%$ and have been responsible for over 10,000 deaths in the U.S. each year (Gershenwald et al. 2017; https://www.cancer.org/cancer/melanoma-skin-cancer/about/keystatistics.html). Although the disease is curable when detected and treated early, the process of differentiating between malignant lesions and the more prevalent benign lesions is challenging. The definitive diagnosis of concerning lesions is achieved through histopathologic assessment of a biopsy specimen, but a considerable rate of discordance even among expert pathologists has been established (Heenan et al. 1984; Boiko et al. 1994; Corona et al. 1996; Farmer et al. 1996; Brochez et al. 2002; Shoo et al. 2010; Gaudi et al. 2013; Niebling et al. 2014; Elmore et al. 2017; Elder et al. 2018). Although accuracy improved following implementation of more defined diagnostic criteria, a large-scale study published by Elmore and colleagues in 2017 reported interobserver discordance rates as high as 57-75\% and intraobserver discordance rates at 37-65\% (Elmore et al. 2017). Together, these observations highlight the complexity and subjectivity of histopathologic assessment and emphasize the need for objective methods for distinguishing malignant from benign lesions to augment current practices.

Molecular biomarkers can provide robust, objective and quantitative measurements of disease state (Rodríguez-Cerdeira et al.; Leachman et al. 2017; Buchbinder and Flaherty 2016). One class of candidate biomarkers is small non-coding microRNAs (miRNAs). Discovered twenty-five years ago (Lee et al. 1993), miRNAs stabilize transcriptional programs (Ebert and Sharp 2012; Judson et al. 2013) and their expression can distinguish cell state transitions during mammalian development and in disease progression (Parchem et al. 2014; Reddy 2015). Combined with a smaller size, reduced complexity, and superior stability over mRNA transcripts, miRNAs are appreciated as potentially valuable candidate biomarkers for many conditions and diseases (Jung et al. 2010; Sheinerman and Umansky 2013). However, despite abundant studies focused on a breadth of diseases, few miRNA biomarkers have 
emerged in the clinical setting (Pogribny 2018). One reason these promising candidate biomarkers have yet to reach their potential is the frequent lack of reproducibility between differential expression studies (Mumford et al. 2018; Nair et al. 2012; Raya et al. 2012; Witwer and Halushka 2016). Discrepancies in differential expression signatures across comparable studies have been attributed to sample heterogeneity, platform-specific biases in miRNA detection, and an absence of standardized normalization strategies (Mumford et al. 2018; Raya et al. 2012; Witwer and Halushka 2016).

Exemplifying these complications are studies that have explored the use of miRNAs as biomarkers for melanoma (reviewed in (Jarry et al. 2014; Jayawardana et al. 2016; Margue et al. 2013; Raya et al. 2012)). Independent studies using various platforms (microarray, RT-qPCR array, RNA-seq) to compare miRNA profiles between benign melanocytic lesions and melanomas have resulted in more than 500 different miRNAs identified as significantly differentially expressed (summarized in Tables S1 \& S2) (Xu et al. 2012; Jukic et al. 2010; Sand et al. 2013; Komina et al. 2016; Kozubek et al. 2013; Hanniford et al. 2015; Latchana et al. 2017; Chen et al. 2011). However, only seven of these miRNAs showed reproducible expression differences in at least half of the cohorts, and none were identified in every study (summarized in Figure S1). Several of the most reproducibly identified miRNAs - miR-211-5p, miR125b-5p, and miR-21-5p - have been validated as differentially expressed in benign and malignant pigmented lesions using in situ hybridization, suggesting some miRNAs could function as biomarkers (Babapoor et al. 2016; Wandler et al. 2017). However, differential expression of these same miRNAs was not observed in 10-30\% of cohorts. Further investigations into the causes of these inconsistencies, and potential solutions, are needed.

In this study, we sought to determine whether a miRNA signature can reliably distinguish malignant from benign melanocytic lesions across both published and independently generated datasets. Machine learning-based classification can help distinguish predictive features from confounding variables, provided the model is trained on an appropriately controlled and annotated dataset (Guyon and Elisseeff 
2003). We generated such a dataset and employed methods of machine learning to both identify the most common confounding variables influencing quantification of miRNA expression from FFPE samples and to generate a refined miRNA signature that correlated uniquely with diagnosis.

\section{Results}

Variation in tumor cell content of FFPE samples confounds miRNA expression analyses

To identify covariates that could confound differential miRNA expression analyses, we took advantage of a cohort of primary melanomas with intact adjacent benign nevi, from which they arose (Shain et al. 2018) (Fig. 1a). The different progression stages for each sample were diagnostically classified by a panel of at least five dermatopathologists and micro-dissected and genotyped for over five hundred cancerrelated genes. Phylogenic trees of the somatic mutations identified in the respective tumor areas were constructed and confirmed the common clonal origin for the different progression stages of each patient. We estimated the tumor cell content (referred to here as tumor cellularity) using allele frequencies and magnitudes of copy number changes as previously described (Shain et al. 2018). Consequently, the dataset was annotated with both clinical features (e.g. patient age, sex, anatomical location of the lesion) as well as genomic information (e.g. mutation burden, copy number variation, tumor cellularity) for each matched pair of nevus and melanoma regions (Fig. 1b and Table S3).

To investigate the influence of each genomic and clinical feature on the miRNA expression pattern, we conducted miRNA sequencing on fifteen of the regions from seven cases (Table S3). In order to first identify potential systemic confounding features, we first employed co-expression analyses for identification of networks of miRNAs sharing similar expression patterns across all regions and identified three co-expression networks (Fig. S2a, Table S4) that were effectively separated via Linear Discriminate Analysis (LDA) (Fig. 1c) (Langfelder and Horvath 2008). Each network consists of miRNAs with read counts that are positively correlated across all samples, regardless of level of expression. We next sought to determine whether the expression patterns of these networks correlated with any of the clinical or 


\section{A. Microdissection of Melanoma and Nevus Areas}

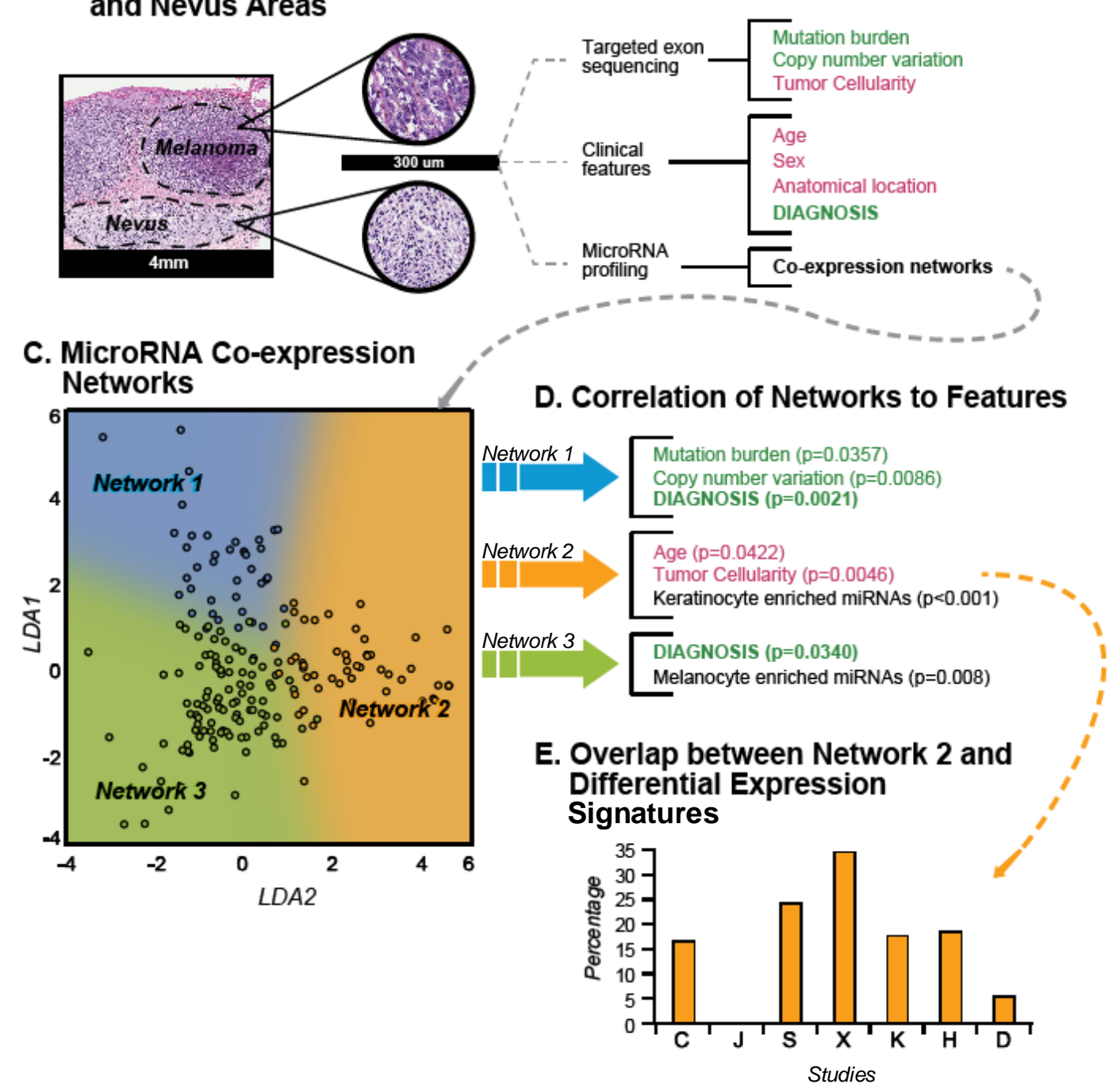

Figure 1:Tumor cellularity and age confound miRNA profiling of melanoma samples A) A cohort of melanomas with intact adjacent precursor nevus were identified and micro-dissected. B) List of features obtained for each micro-dissected region either from targeted exon sequencing (genetic features) or the pathology requisition form (clinical features). Red font indicates potentially confounding features. Green font indicates target feature (diagnosis) or genetic features highly correlated with target feature. C) miRNA co-expression networks. Scatter plot of all expressed miRNAs separated by LDA trained on the three networks. The three coexpression networks are indicated by the color of the points (blue, orange, and green). D) Correlations of PC1 of each miRNA network expression matrix with clinical and genetic features from (B) with p-values calculated from the corresponding correlation coefficient. E) Percent of miRNAs identified as differentially expressed between nevi and melanoma in seven published studies that overlap with Network 2. Published studies are identified by the first letter of the first authors' last names (Table S1).

genomic annotations of the samples, including not only diagnosis but also potentially confounding features, such as patient age. We summarized the miRNA expression matrix for each network by its first principal component and compared these to the sample covariates (Fig. 1d, Fig. S2b-c). Two of the networks (Network 1 and Network 3) were significantly correlated with a diagnosis of melanoma and 
were not influenced by tumor cellularity or any other clinical feature. Network 1 was also correlated with mutation burden and copy number variation, both measurements of genome damage that increase during progression from nevus to melanoma (Shain et al. 2015).

In contrast to the two melanoma-associated networks, Network 2 was positively correlated with tumor cellularity and, to a lesser extent, patient age. This observation suggests that although miRNAs within Network 2 were differentially expressed in melanoma and nevus samples, variation in their observed abundance may reflect the extent of contamination with non-tumor cells rather than different progression stages. Consistent with this interpretation, we observed that miRNAs known to be expressed in cultured primary human keratinocytes were enriched in Network 2 as would be expected if keratinocytes were a significant fraction of contaminating non-tumor cells (Fig. 1d, Fig. S2c). Conversely, miRNAs known to be expressed in cultured primary human melanocytes were enriched in Network 3 consistent with changes in Network 3 reflecting melanocyte biology. Together, these data suggest that miRNA profiling datasets derived from micro-dissected FFPE samples can contain sufficient levels of contaminating non-tumor cells to influence the overall miRNA expression profile. Contamination by non-tumor cells is expected to vary among samples dependent on their size, histologic type (predominantly junctional versus intradermal), and preparation (e.g. precision of microdissection). If not controlled for, variation in tumor cellularity is expected to degrade the reproducibility of signatures across studies. Indeed, miRNAs from Network 2 constituted up to thirty percent of the miRNAs in expression signatures reported from the seven previously reported datasets (Fig. 1e). This result highlights the need for alternative analytical methods for identifying miRNA signatures predictive of melanoma diagnosis from FFPE derived samples.

\section{Classification of nevus from melanoma samples with miRNA ratios}

To identify miRNAs that best distinguish malignant from benign melanocytic lesions, we utilized feature selection (FS). FS is a method of machine learning that is frequently used to simplify predictive models 
and to avoid analytical pitfalls such as the phenomena of over-fitting and the 'curse of dimensionality' (He and Yu 2010; Saeys et al. 2007). In the context of biology, FS methods can be applied to gene expression datasets to identify sets of features (in this case, miRNAs) that are more often biologically relevant and ultimately improve classification performance (Abeel et al. 2010). There are common FS methods, such as univariate statistical test filtering (e.g. FDR, t-test) and feature rank wrappers (e.g. backward selection) that will identify individual features that are independently relevant, but they miss features that are only relevant in the context of complex networks (Mnich and Rudnicki 2017; Wenric and Shemirani 2018). An alternative strategy is the all-relevant features (ARF) selection approach that involves multiple iterations of feature ranking and can determine both weak and strong relevant features (Kursa and Rudnicki 2010). An ARF method called Boruta (Fig. S3) has been shown to provide improved performance in a variety of datasets including gene array data and environmental science data (Li et al. 2016; Kursa 2014).

We employed Boruta to obtain an initial list of those miRNAs that were most important for discriminating the benign and malignant regions from our cohort across 1000 random forest iterations (Fig. 2a). All miRNAs with more than five total reads were considered, resulting in 341 unique features. For each miRNA, a second artificial feature was generated through randomized re-distribution of the read counts across samples (Fig. S3). These 'shadow features' provided an equal number of negative control features for which to compare each experimental feature. We conducted Boruta with the combined 682 experimental and negative control features, ranking the importance of each feature for the accurate classification of nevus samples from melanoma samples with each iteration. We identified 38 miRNAs that ranked higher than the maximum-performing shadow feature with a p-value of less than 0.001 (Fig S4). To enable comparison with published studies we also required that the expression levels of each miRNA were assessed in all published datasets (Table S2). The final list of feature-selected miRNAs contained two miRNAs with increased expression (miR-31-5p, miR-21-5p) and four miRNAs (miR-211- 
bioRxiv preprint doi: https://doi.org/10.1101/507400; this version posted December 27, 2018. The copyright holder for this preprint (which was not certified by peer review) is the author/funder, who has granted bioRxiv a license to display the preprint in perpetuity. It is made available under aCC-BY-NC-ND 4.0 International license.

\section{A. miRNA Classification Workflow B. Boruta Feature Selection}

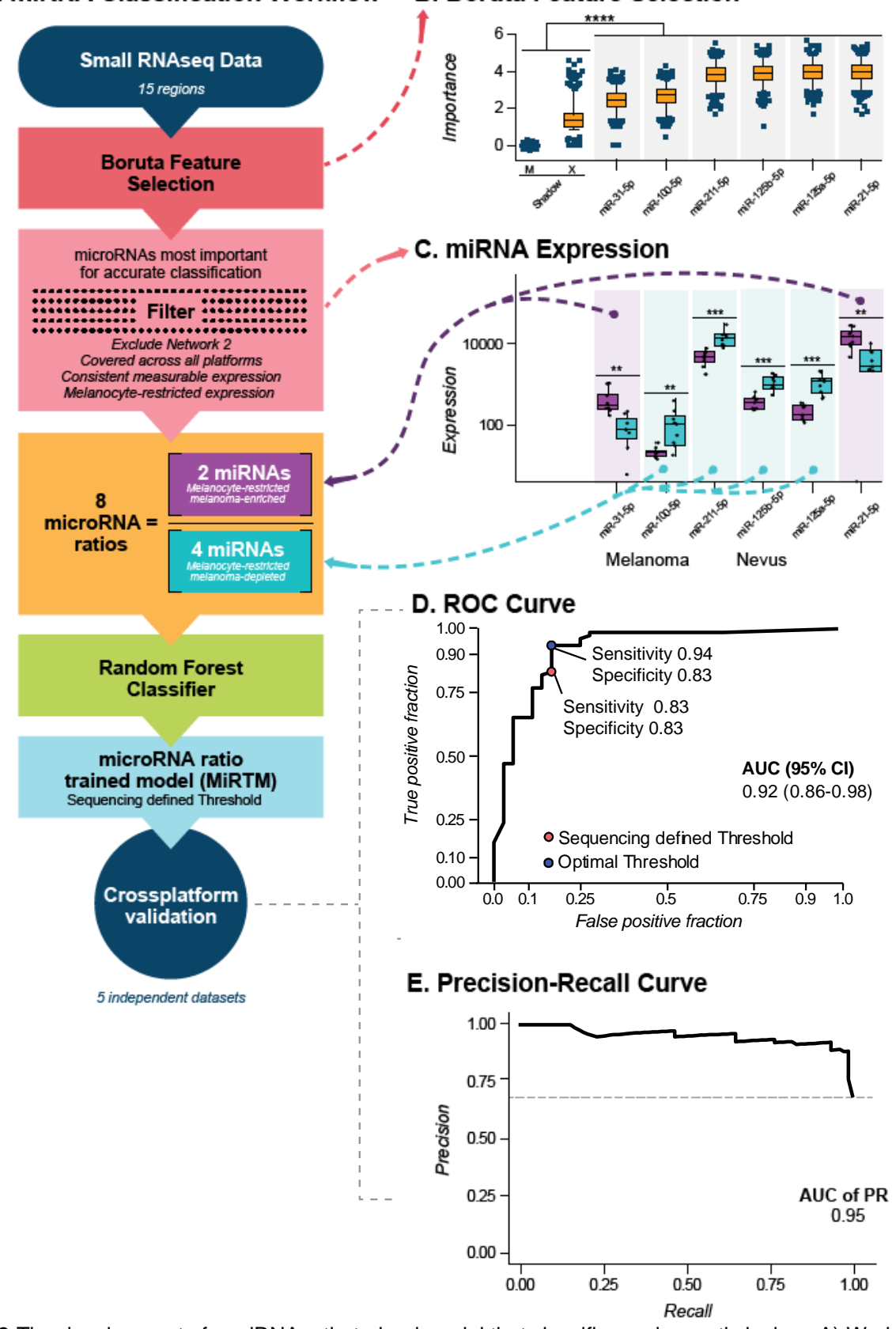

Figure 2:The development of a miRNA ratio-trained model that classifies melanocytic lesions A) Workflow of miRNA-Seq classifier development and testing. B)Top miRNAs for classifying nevi from melanomas using Boruta feature selection. Each feature-selected miRNA has a significantly higher importance value than the random shadow max feature $(X)$ and shadow mean feature (M) (Fig. S3). The top six miRNAs are shown (full list in Fig. S4). C) Normalized miRNA-Seq counts from micro-dissected FFPE samples. Counts from melanoma regions (purple) and nevus regions (blue) are shown. Boxes indicate mean, first and third quartiles. miRNAs designated as melanoma-enriched or melanoma-depleted are designated by light purple and light blue backgrounds, respectively. D) ROC curves for cross-platform testing of MiRTM using the combined set of publicly available datasets. Threshold determined from the discovery sequencing set and optimal threshold are shown in as red and blue points respectively with corresponding sensitivity and specificity annotated for each. E) Precision-Recall curve for testing of the combined set using MiRTM. 
5p, miR-125a-5p, miR-125b-5p, miR-100-5p) with decreased expression in melanomas (Fig. 2c). These miRNAs are referred to as melanoma-enriched and melanoma-depleted miRNAs, respectively.

The use of transcript ratios has been previously demonstrated to strengthen the prediction accuracy through simplification of features (Avissar et al. 2009; Reddy et al. 2015). We developed a diagnostic score using all ratios of melanoma-enriched miRNAs to melanoma-depleted miRNAs (Fig. 2a). This approach controls for variations in lesion composition (e.g. the relative amount of malignant and benign tissue) when micro-dissection boundaries are not known and differences in tumor cellularity when genetic data are not available. This approach also normalizes for the fraction of miRNAs of melanocytic origin (as opposed to the totality of miRNAs), amplifies the signal from malignant cells by normalizing melanoma-enriched miRNAs to melanoma-depleted miRNAs, and permits cross-platform comparisons without the need for cross-platform normalization. We divided each of the two melanoma-enriched miRNAs by each of the four melanoma-depleted miRNAs, producing eight miRNA ratios (miR-31-5p/ miR-211-5p, miR-31-5p/ miR-125a-5p, miR-31-5p/ miR-125b-5p, miR-31-5p/ miR-100-5p, miR-21-5p/ miR-211-5p, miR-21-5p/ miR-125a-5p, miR-21-5p/ miR-125b-5p, and miR-21-5p/ miR-100-5p). These eight ratios were used to train a random forest classifier. The final miRNA Ratio Trained Model (MiRTM) resulted in an area under receiver operating characteristic curves (AUC) of 1.0 for the discovery set of 7 samples containing 15 matched melanoma and nevus regions.

\section{Validation of the MiRTM on previously published datasets}

To test the accuracy of the MiRTM on independent datasets we obtained and combined the raw data from five previously published miRNA profiling studies (Figs. S1, Table S1; Sand, Xu, Chen, Komina \& Jukic). These studies contained both microarray and RT-qPCR datasets. Regardless of the platform, the eight miRNA expression ratios were used as input for the model trained on the sequencing data. The MiRTM resulted in an AUC of ROC of 0.92 (Fig. 2d) and an AUC of Precision-Recall of 0.95 (Fig. 2e). We set two thresholds to calculate sensitivity and specificity. First, we used the optimal threshold as 
defined by our sequencing cohort (0.5), which resulted in a sensitivity of 0.83 and a specificity of 0.83

(Fig. 2d, red point). As this validation cohort was constructed using different technical platforms for miRNA profiling, we also calculated sensitivity and specificity using the optimal threshold for the ROC curve as 0.94 and 0.83 respectively (Fig. 2d, blue point).

To further examine the reproducibility of our model, we ran MiRTM on each individual previously published dataset. Across all datasets, the MiRTM resulted in an AUC of 0.8 or higher, with an average of 0.922 (Fig. S5a). Optimal sensitivity remained above 0.9 across each dataset (Fig. S5b). With the exception of one dataset, optimal specificity was above 0.8 (Fig. S5b). Interestingly, that dataset (Fig. S5, X) contained the highest level of the miRNA network we identified as associated with tumor cellularity (Fig. 1e, X).)

\section{Validation of the MiRTM on randomly selected cases}

Discovery phase cohorts are often selected for unambiguous and homogenous cases. To further validate our model on a greater diversity of cases, we randomly retrieved 82 biopsied melanocytic lesions - 41 neoplasms diagnosed as nevi and 41 diagnosed as melanoma - from the archives of the UCSF Dermatopathology Section. All diagnoses were reviewed and confirmed by an independent dermatopathologist. This cohort contained a greater range of tumor cellularity and subtypes of melanocytic neoplasms than the discovery cohort (Table 1, Table S5). Instead of micro-dissection, entire FFPE sections were scraped to obtain bulk RNA. The abundance of the six miRNAs was assessed by RTqPCR (Fig. 3a). We converted $C_{t}$ values to expression ratios using the linear transformation $\left(2^{\wedge}-C_{t}\right)$ and ran MiRTM. The resultant AUC of ROC for our unfiltered cohort (UC) was 0.92 (Fig. 3b). The AUC of the Precision-Recall curve was 0.911 (Fig. 3c). To classify the lesions into benign and malignant, we again considered two thresholds. Using the threshold defined by our sequencing cohort (0.5) the model 
achieved sensitivity of 0.83 and specificity of 0.71 (Fig. $3 \mathrm{~b}$ red point). The optimal sensitivity and specificity of this ROC curve were 0.81 and 0.90 (Fig. $3 \mathrm{~b}$ blue point).

Table 1: Sample information for unfiltered cohort

\begin{tabular}{|c|c|c|c|}
\hline Features & & $\begin{array}{c}\text { Melanoma } \\
\mathrm{n}(\%)\end{array}$ & $\begin{array}{l}\text { Nevus } \\
n(\%)\end{array}$ \\
\hline & Totals & $41(100)$ & $41(100)$ \\
\hline \multirow{3}{*}{ Age } & $<30$ & $0(0)$ & $6(15)$ \\
\hline & $30-60$ & $21(51)$ & $28(68)$ \\
\hline & $>60$ & $20(49)$ & $6(15)$ \\
\hline \multirow{3}{*}{ Stage } & $\mathrm{pT} 1 \mathrm{a} / \mathrm{b}$ & $35(85)$ & - \\
\hline & $\mathrm{pT} 2 \mathrm{a} / \mathrm{b}$ & $3(7)$ & - \\
\hline & pT34 a/b & $2(5)$ & - \\
\hline \multirow{3}{*}{$\begin{array}{l}\text { Melanoma } \\
\text { Subtype }\end{array}$} & SSM & $33(80)$ & - \\
\hline & Nodular & $4(10)$ & - \\
\hline & NoS & $3(7)$ & - \\
\hline \multirow{4}{*}{$\begin{array}{l}\text { Nevus } \\
\text { Subtypes }\end{array}$} & Congenital & - & $15(37)$ \\
\hline & Compound & - & $32(78)$ \\
\hline & Lentiginous & - & $26(63)$ \\
\hline & Dysplastic & - & $7(17)$ \\
\hline \multirow{3}{*}{$\begin{array}{c}\text { Percent } \\
\text { Tumor Cell }\end{array}$} & $<40$ & $13(32)$ & $21(51)$ \\
\hline & $40-70$ & $19(46)$ & $15(37)$ \\
\hline & $>70$ & $13(32)$ & $5(12)$ \\
\hline Inflammation & Yes & $28(68)$ & $5(12)$ \\
\hline \multirow{3}{*}{$\begin{array}{l}\text { Percent } \\
\text { Melanoma } \\
\text { vs Nevus }\end{array}$} & $<80$ & $3(7)$ & - \\
\hline & $80-100$ & $10(24)$ & - \\
\hline & 100 & $28(69)$ & - \\
\hline
\end{tabular}

As the MiRTM demonstrated a lower sensitivity for the more diverse second validation cohort compared to the previously published cohorts, we examined whether the model was affected by tumor cellularity and patient age. When we compared the MiRTM score to the tumor cellularity in each sample as assessed by a dermatopathologist, we found no correlation (Fig. 4a), suggesting that the MiRTM score was unaffected by this variable. Similarly, we found no correlation between MiRTM score and age (Fig. S6). To investigate whether other features might influence the MiRTM score, we calculated the correlations between fourteen clinical features and the 


\section{A. Unfiltered Validation Cohort}

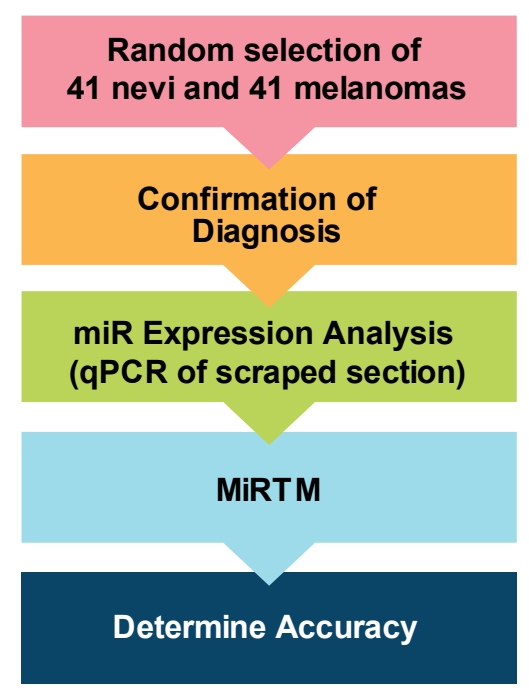

\section{B. ROC Curve}

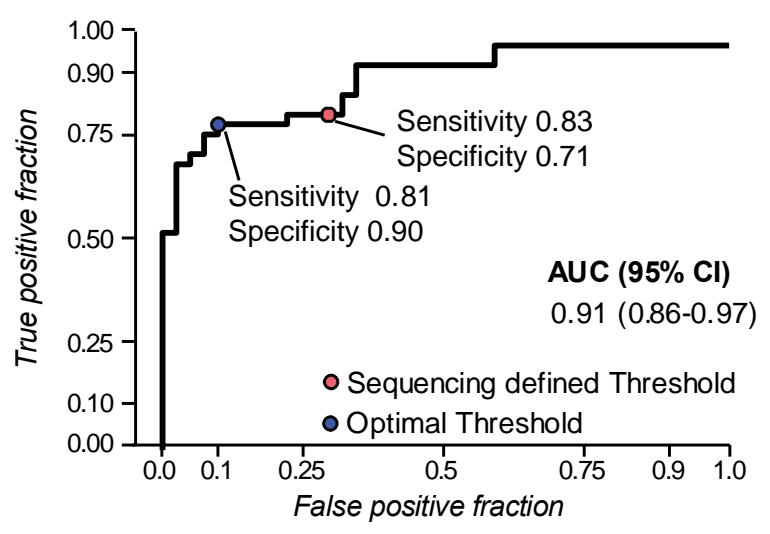

C. Precision-Recall Curve

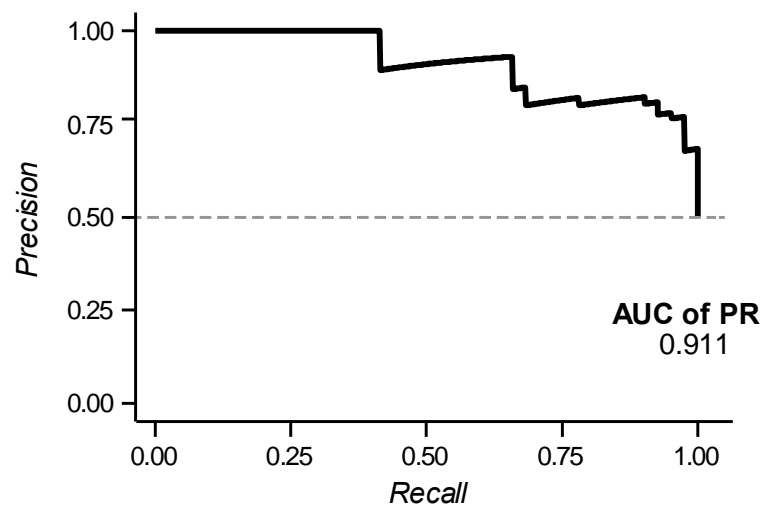

Figure 3: MiRTM validation

A) Workflow for assembling and testing a randomly selected cohort of melanomas and nevi for the validation cohort. B) ROC curve for validation cohort using MiRTM. Threshold determined from the discovery sequencing set and optimal threshold are shown in as red and blue points respectively with corresponding sensitivity and specificity annotated for each. C) Precision-Recall curve for testing of the validation cohort using MiRTM.

MiRTM score (Fig. 4b-c, Table S5). In the melanoma samples, we observed the expected correlations between clinical features such as age and solar elastosis as a proxy for mutation burden (Fig. 4b).

However, we observed no significant correlations with the MiRTM score that suggested any of the features other than diagnosis could influence the score. For example, changes in the thickness or size of the lesion did not affect the MiRTM score. Similarly, in the nevus samples, most features did not influence the score, including the presence of dysplastic features (Fig. 4c). However, exclusively among the benign lesions, the MiRTM score was positively correlated with inflammation (Fig. 4c-d). Together, these data demonstrate that while the MiRTM score is robust against heterogeneity in lesion size, tumor 


\section{A. Correlation with Tumor Cellularity}

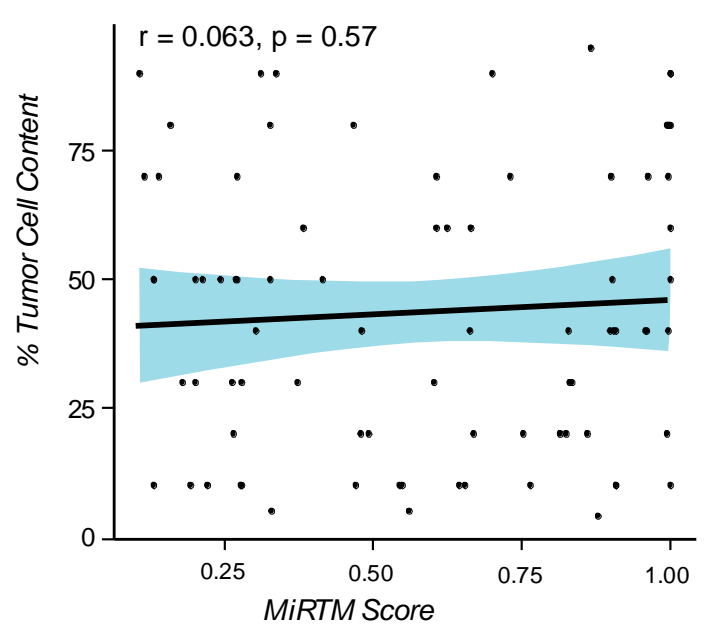

C. MiRTM Correlation Matrix (Nevus )

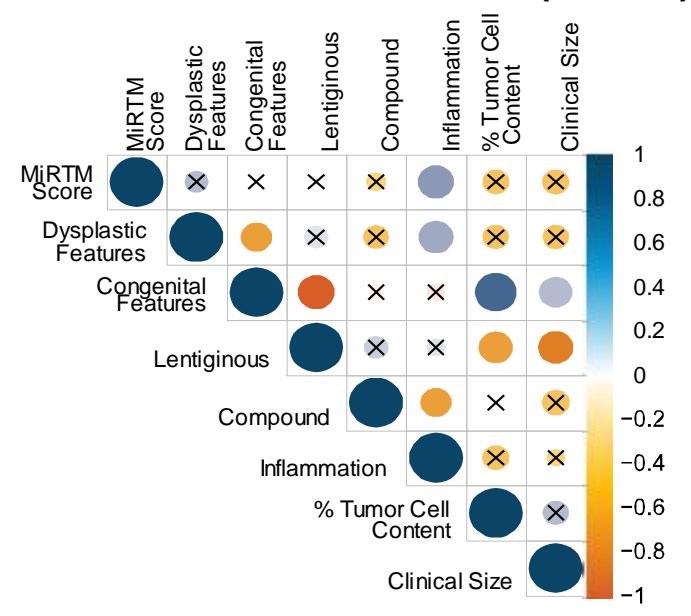

B. MiRTM Correlation Matrix (Melanoma'

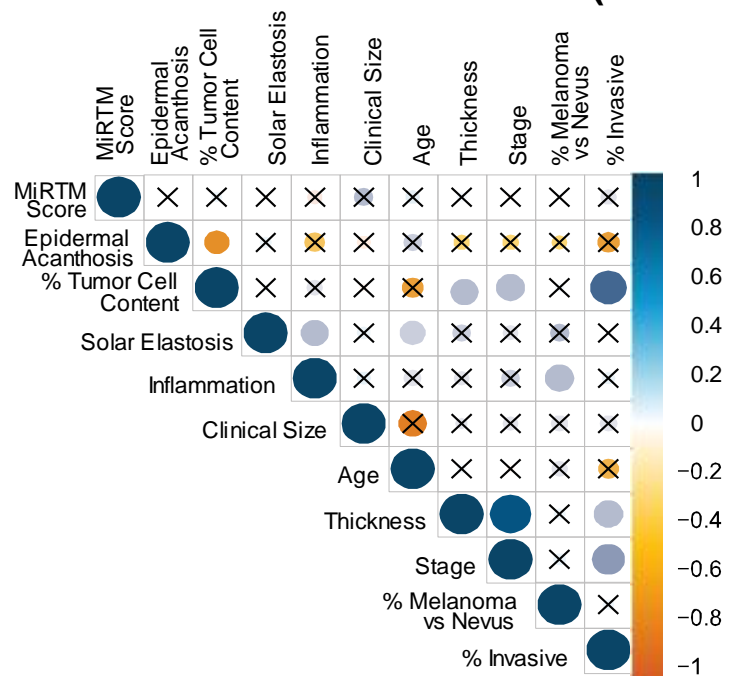

D. hflammation Association

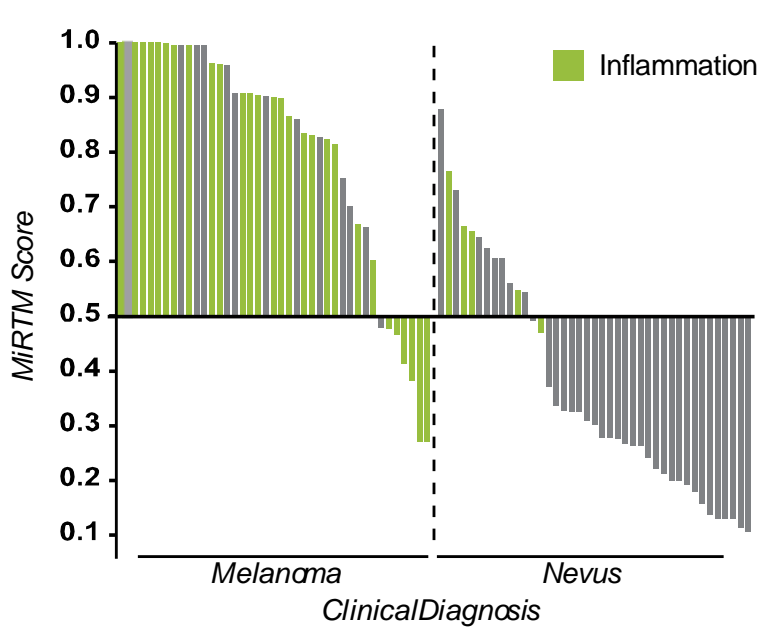

Figure 4: MiRTM accuracy is unaffected by tumor cellularity A) Correlation of MiRTM score with percent tumor cell content. $95 \% \mathrm{Cl}$ is shown in blue. $\mathrm{B}-\mathrm{C}$ ) Correlation matrix for histological and clinical features with MiRTM score for melanoma (B) and nevus (C) samples. Non-significant correlations are shown with an X, while direction and size of significant correlations are indicated by color. D) MiRTM scores for each sample in the validation cohort. Scores above 0.5 were called malignant. Samples microscopically graded as inflamed are colored in green.

cellularity, and presence of dysplastic features, the presence of inflammatory cells might result in false positive calls.

\section{Discussion}


Numerous studies have analyzed miRNA expression at different stages of melanoma progression, collectively identifying over 500 miRNAs enriched in nevi or melanomas (Babapoor et al. 2017; Kozubek et al. 2013; Hanniford et al. 2015; Jukic et al. 2010; Satzger et al. 2012; Sand et al. 2013; Xu et al. 2012; Komina et al. 2016; Chen et al. 2011; Latchana et al. 2017). Our analyses have refined this expansive list to six miRNAs that reproducibly distinguish nevi from melanoma across independent datasets and profiling platforms. We identified this signature by controlling two important variables, interobserver variability of diagnosis and variability in tumor cellularity. To address the first variable, we utilized a discovery cohort for which the diagnosis accuracy was established by requiring the median concordance among five to eight different dermatopathologists and supporting genetic features, eliminating the possibility of training the model with misdiagnosed cases. Secondly, melanomas and nevi were matched as pairs representing different progression stages of the same neoplastic clone, eliminating variability by comparing different lesions from different patients. Thirdly, we identified and excluded miRNAs from the signature, whose expression is influenced by tumor cellularity, thereby removing a covariate that has confounded previous analyses. When tested on six datasets assembled by independent groups, a model trained on expression ratios of the refined signature classified benign from malignant melanocytic lesions with an average AUC of ROC above 0.91.

Our strategy required a meticulously assembled and annotated initial cohort of lesions and next generation small RNA sequencing. Although this approach permitted us to identify confounding covariates, the cost and effort to obtain each case also constrained the size of the training cohort to only fifteen samples. One fundamental feature of machine learning is that increased training improves accuracy. Thus, the current performance of the model represents the lower bound of the potential accuracy that could be obtained with a larger training of similar cases. Despite the limited size of the training set, the sensitivity and specificity of the MiRTM for validation sets thresholded on the discovery cohort was 0.83 and $0.71-0.83$. This performance of the MiRTM is comparable to other molecular tests for distinguishing benign melanocytic nevi from melanoma, including chromosomal analysis by fluorescence in situ hybridization 
(sensitivity 0.72-1.00, specificity 0.90-1.00) (Gerami et al. 2010; Ferrara and De Vanna 2016) and myPath Melanoma gene expression profiling (sensitivity $0.63-0.90$, specificity $0.88-0.93$ )(Clarke et al. 2017; Minca et al. 2016). The MiRTM does not perform as well as chromosomal analysis by array comparative genomic hybridization (aCGH, sensitivity $0.92-0.96$, specificity $0.87-1.00$ )(Bastian et al. 2003; Wang et al. 2013). However, assessment by the MiRTM requires only a single section of FFPE material, does not require microdissection and RT-qPCR is a quick and affordable assay making this approach a candidate for lesions where tissue availability is limited.

It is important to note that our discovery set and validation sets were obtained using diverse platforms of miRNA profiling. Indeed, the threshold for optimal thresholds for both validation sets (microarray and RT-qPCR) were slightly different than the optimal threshold for the discovery set (small RNA sequencing). Although the over-all crossplatform performance demonstrates the robustness of the model, future studies aimed at clinical development of this model should consider training and validating the model on a single platform.

The only measured feature that correlated with the MiRTM score overall was diagnosis. However, in the benign samples of the validation set, we observed that inflammation can result in false-positive calls. Indeed, some of the miRNAs (miR-125b, miR-31, miR-21) have been associated with inflammation in psoriasis (Hawkes et al. 2016). However, inflammation was not correlated with the MiRTM score over all samples in the cohort suggesting the feature-selected miRNAs are not exclusively an inflammation signature. Further training on a larger cohort selected for differential inflammation status could substantially reduce the number of false positives.

Of the six miRNAs of our signature, three (miR-211-5p, miR-21-5p, and miR-125b-5p) (Fig. S1a) have been linked to melanoma, have been previously validated by in situ hybridization (Babapoor et al. 2016; Latchana et al. 2016), and have been functionally assessed in melanoma cell lines. MiR-21 is an 
established oncomir and regulates genes involved in increased proliferation and invasion (Satzger et al. 2012). It is upregulated in many cancers including melanoma and its expression correlates with progression from nevi to primary melanomas and then to metastatic melanomas (Satzger et al. 2012; Jiang et al. 2012). Conversely, miR-125b is often downregulated in cancers, including advanced melanomas, where its loss results in increased expression of cJUN and MLK3 (Zhang et al. 2014; Kappelmann et al. 2013). MiR-211 is among the most well-established functional miRNAs in melanocytes and is downstream of the important melanocyte lineage transcription factor MITF (Mazar et al. 2010). It is often downregulated during melanoma progression and has been linked to invasion through regulation of BRN2, NFAT5 and TGFßR2 (Levy et al. 2010; Boyle et al. 2011). The other miRNAs in the signature are less well characterized in melanocytes. As another miR-125 family member, miR-125a is expected to target a similar set of genes as miR-125b, but has been mostly described in other cancers. MiR-31 is upregulated in some cancers, but its role as an obligate oncomir is controversial as it is transcribed from a commonly deleted or methylated genomic region in many cancers (Valastyan and Weinberg 2010; Asangani et al. 2012). Similarly, miR-100 has also been described as both a tumor suppressor and an oncomir depending on the context (Li et al. 2015). Regardless of their precise functional role in the context of melanocytic neoplasia, our analyses demonstrate that the relative expression ratios of these six miRNAs can assist in distinguishing benign melanocytic nevi from malignant melanoma in FFPE samples.

\section{Methods}

Meta-analysis

For meta-analyses summarized in Fig. S1, we used all datasets in public databases that contained miRNA profiling for both primary melanoma and nevus samples for comparison to our knowledge (Table S1) (GSE19229, GSE36236, GSE24996, GSE62372, GSE35579, GSE34460, and E-MTAB-4915). The top differentially expressed miRNAs for each dataset were determined using an FDR cutoff of 0.05 using either Limma (microarray and TaqMan array data (Ritchie et al. 2015)) or DeSeq2 (miRNA-seq data 
(Love et al. 2014)). To determine overlap (Fig. S1a), only those miRNAs for which probes were included in every detection platform were considered (Table S2). Overlap was plotted using the UpsetR package in R (Conway et al. 2017).

Clinical specimens and histopathologic assessment

A training cohort of melanomas with an intact adjacent benign nevus constituted the discovery cohort for this study. Fifteen different areas ( 8 malignant and 7 benign) from seven cases were selected based upon which samples from a larger published cohort that was previously genetically assessed (Shain et al. 2018) had leftover material. All cases had previously been retrieved from the UCSF Dermatopathology archive as formalin-fixed paraffin-embedded (FFPE) tissue blocks. Histopathologically distinct areas had been independently evaluated by a panel of 5-8 dermatopathologists for staging (Shain et al. 2018). Distinct tumor areas were manually micro-dissected with a scalpel under a dissection scope from unstained tissue sections following the guidance of a pathologist in order to limit stromal cell contamination. Previously, genetic DNA had been isolated from four $10 \mu \mathrm{M}$ sections using Qiagen DNA FFPE Tissue Kit (Cat\# 56404) (Shain et al. 2018). For this study, four additional 20uM sections were dissected and total RNA was isolated using the RecoverALL Total Nucleic Acid Isolation Kit for FFPE (Ambion). An independent validation cohort was generated by retrieving obtaining 82 diagnosed melanomas (41 cases) or nevi (41 cases) from UCSF Dermatopathology. Cases were reevaluated by a separate dermatopathologist to confirm diagnosis and obtain histopathological features, but were not excluded for any reason. For RNA isolation of the test cohort, one $20 \mu \mathrm{M}$ section was scraped off the slide and processed in its entirety without micro-dissection and total RNA was isolated using the RecoverALL Total Nucleic Acid Isolation Kit for FFPE (Ambion).

MicroRNA-seq and analysis

MicroRNA sequencing libraries were constructed with the TailorMix Small RNA Library Preparation Kit (SeqMatic, CA) using total RNA extracted from FFPE samples. Sequencing was performed on the 
Illumina HiSeq2500 platform at single-end 50bp. After adaptor sequences were removed, reads were aligned to a human reference (hg37) with Bowtie (Langmead et al. 2009) and then small RNA reference groups (miRBase21) were counted. Data were submitted to dbGaP (phs001550.v2.p1). Differential expression analysis was performed from feature counts using DeSeq2 (Love et al. 2014) with p-values adjusted for multiple testing with the Benjamin-Hochberg method (p-adj).

\section{Co-expression Analysis}

Co-expression analysis was restricted to 805 miRNAs with at least one read in at least two samples. Three co-expression networks were identified in R using a four-step approach as previously described (Lui et al. 2014) and a minimum 10-member seed and 0.85 correlation threshold. First, pairwise biweight midcorrelation coefficients (cor) were calculated for all possible pairs of miRNAs for all samples. Second, miRNAs were clustered using the flashClust implementation of a hierarchical clustering procedure with complete linkage and 1 - cor as a distance measure (Langfelder and Horvath 2008). The resulting dendrogram was cut at a static height of $\sim 0.48$, corresponding to the top $10 \%$ of pairwise correlations for the entire dataset. Third, all clusters consisting of at least 10 members were identified and summarized by their eigengene (i.e. the first principal component obtained via singular value decomposition of the standardized miRNA expression matrix corresponding to each initial cluster) (Horvath and Dong 2008). Fourth, highly similar networks were merged if the Pearson correlation coefficients of their eigengenes exceeded 0.85 . This procedure was performed iteratively such that the pair of networks with the highest correlation $>0.85$ was merged, followed by recalculation of all eigengenes, followed by recalculation of all correlations, until no pairs of networks exceeded the threshold. Following these steps, three co-expression networks were identified. The strength of association $\left(k_{\mathrm{ME}}\right)$ between each miRNA and each network was determined by calculating the Pearson correlation between its expression pattern over all samples with each eigengene (Horvath and Dong 2008) (Table S4). 


\section{Linear Discriminant Analysis}

To assess and visualize the degree to which the three different miRNA groups can be distinguished based on their expression in our samples, we constructed a classifier that predicts the miRNA network. We used Linear Discriminant Analysis to project the expression of miRNAs in each sample into a two-dimensional linear subspace that optimally separates the different miRNA categories. We subsequently trained a support vector machine with the linear kernel to distinguish between the miRNA categories. An area under the ROC curve for distinguishing blue, green and orange categories (Fig. 1c) in a testing set was $0.97,0.94$, and 0.95 , respectively.

Gene set enrichment analysis

GSEA was conducted using the three co-expressed miRNA networks as gene sets against public miRNA expression datasets (GSE16368) comparing either primary human melanocytes (GSM817251

GSM1127159, GSM1127164) to keratinocytes (GSM817253, GSM1127111, GSM1127113) or primary human melanocytes to fibroblasts (GSM817252, GSM1127116). Positive enrichment of each case corresponded to melanocyte-enriched and negative corresponded to either fibroblast- or keratinocyteenriched.

\section{Genomic Analysis}

The targeted exon sequencing datasets for each sample of the training cohort were accessed from dbGaP (phs001550.v1.p1). Read alignment, mutational analysis, and copy number analysis was performed as previously described (Shain et al. 2015; Talevich et al. 2016). Briefly, sequences were aligned using Burrows-Wheeler Aligner (BWA) (Li and Durbin 2009) with mutational analysis and processing performed using Picard and Genome Analysis Toolkit. Copy number information was obtained with the use of CNVkit. Tumor cell content (tumor cellularity) was calculated bioinformatically using multiple methods when possible, including median mutant allele frequency (MAF) of somatic mutations, MAF of 
the driver mutation, allelic imbalance over germline and others as previously described (Shain et al. 2018).

\section{Classifier analyses}

A feature subset was selected using the Boruta R package (Kursa and Rudnicki 2010) to determine a minimal set of miRNAs for classifier predictive accuracy from the FFPE miRNA-seq data set. Briefly, for each feature (miRNA) present in the RNA-seq data set, a control "shadow-feature" (shadow miR) of comparable expression and variance was generated through random re-assignment of the read counts to different samples (Fig. S3). The combined feature set (miRNAs and shadow miRs) was used to train a random forest classifier and the importance of each feature for the accuracy of the model was determined. This process was repeated in 1000 iterations, with miRNAs excluded from each ensuing round once they were significantly less important than the maximum important shadow miR. Thus, the remaining list of miRNAs at the conclusion of the analyses represents only those miRNAs that out-performed an equal number of randomized controls by a statistically significant margin. This initial miRNA list was then further refined by removing miRNAs that were below a minimum expression threshold and/or were not detected across all outside test sets to obtain a final list of 6 miRNAs (miR-211-5p, miR-125a-5p, 125b5p, miR-100-5p, miR-31-5p and miR-21-5p). Using log fold-change information from differential expression analysis, each miRNA was associated as melanoma-enriched (ME) or melanoma-depleted / nevus-associated (MD) and miRNA ratios were created from each combination of the 2 ME miRNA (miR-31-5p and miR-21-5p) and 4 MD miRNA (miR-211-5p, miR-125a-5p, 125b-5p, miR-100-5p). A random forest classifier was then built using this transformed minimal signature set and tested by 5 -fold repeated cross-validation over 100 repeats to create a final miRNA ratio trained model (MiRTM). The MiRTM was used to classify the datasets described in the meta-analysis where sufficient data were available to obtain sensitivities, specificities, and overall performance by AUC through a ROC curve for each set (Fig. S5) or combined as a group (Fig. 2d-e). The Dadras and Hernando datasets were omitted from the analysis due to insufficient sample sizes (Dadras contained 2 nevus samples), or too many 
missing features and large sample imbalance (Hernando contained a nevus/mel ratio of 0.1 and many features removed in processing) (Kozubek et al. 2013; Hanniford et al. 2015). Similarly, MiRTM was used to classify each case in our validation set with sensitivities and specificities determined using either an optimal threshold based on the Youden index or the sequencing determined threshold 0.5 (Fig. 3b). Overall performance was visualized by the area under a ROC or precision recall curve respectively (Fig. $3 b-c)$.

miRNA qPCR assay

Total RNA was converted into cDNA using the TaqMan Advanced miRNA cDNA Synthesis Kit (Thermo Fisher A28007) following the manufacturer recommended protocols. Quantitative PCR for specific miRNA detection was conducted with TaqMan Advanced miRNA Assays (Thermo Fisher) using the TaqMan Fast Advanced Master Mix (Thermo Fisher 4444557) and analyzed on the Applied Biosystems 7900HT instrument following recommended protocols.

\section{Statistical Analysis}

Statistical significance was set to 0.05 with p-values adjusted for multiple testing with the BenjaminHochberg method. Pearson correlation coefficients were obtained between all continuous features with the equivalent point biserial correlation coefficient for binary variables. Correlation matrices were plotted with the corrplot R Package and correlation plots with the ggpubr R package with $95 \%$ confidence intervals calculated for the curves. Sensitivities and specificities were calculated from classification models built using the caret $\mathrm{R}$ package. ROC curves were generated using the pROC R package. Confidence intervals (CI) were calculated from the 95\% CI of 2000 bootstrap replicates for sensitivity and specificity or the 'Delong' method for AUCs using pROC R package. The precision-recall plot was generated using the precrec $\mathrm{R}$ package. All data was processed in $\mathrm{R}$ (3.3.2)

\section{Data Access}


The sequrncing data was submitted to the NCBI database of Genotypes and Phenotypes (dbGaP) accession number phs001550.v2.p1.

\section{Acknowledgements}

This research was supported in part by NIH DP5OD019787 to R.L.J., the Sandler Foundation Program for Breakthrough Biomedical Research Fellowship to R.L.J., the Marcus Program in Precision Medicine Innovation Fund Seeding Big Ideas Award to R.L.J and M.L.W. and the Helen Diller Family Comprehensive Cancer Center Impact Award to M.L.W. and R.L.J. We would like to thank Tim H. McCalmont for assistance in identifying cases and Hilary Faith Hickman for assistance in visual design.

\section{Author Contributions.}

R.T. conducted meta-analyses, gene set enrichment analyses, feature selection and training of classifiers. M.H. performed the LDA. S.J.S and M.C.O. performed the co-expression network analyses. Cohort 1 was assembled by A.H.S. I.Y., B.C.B. and N.M.J and micro-dissected by N.M.J and R.L.J. R.L.J. conducted RNA and DNA isolation. A.H.S. performed genetic analyses. Cohort 2 samples and features were assembled by U.E.L. R.T. conducted RNA isolation, RT-qPCR, and analyses. U.E.L., N.M.J, I.Y., and B.C.B. provided histopathology and diagnoses. R.T., I.Y., M.L.W., M.C.O., B.C.B and R.L.J provided significant intellectual contribution and critical reading of the manuscript. R.L.J. conceived of and directed all experiments.

\section{References}

Abeel T, Helleputte T, de Peer Y Van, Dupont P, Saeys Y. 2010. Robust biomarker identification for cancer diagnosis with ensemble feature selection methods. Bioinformatics 26: 392-398.

Asangani IA, Harms PW, Dodson L, Pandhi M, Kunju LP, Maher CA, Fullen DR, Johnson TM,

Giordano TJ, Palanisamy N, et al. 2012. Genetic and epigenetic loss of microRNA-31 leads to feedforward expression of EZH2 in melanoma. Oncotarget 3: 1011-1025.

Avissar M, Christensen BC, Kelsey KT, Marsit CJ. 2009. A MicroRNA Expression Ratio is Predictive of 
Head and Neck Squamous Cell Carcinoma. Clin Cancer Res 15: 2850-2855.

Babapoor S, Horwich M, Wu R, Levinson S, Gandhi M, Makkar H, Kristjansson A, Chang M, Dadras

SS. 2016. microRNA in situ hybridization for miR-211 detection as an ancillary test in melanoma diagnosis. Mod Pathol 29: 461-475.

Babapoor S, Wu R, Kozubek J, Auidi D, Grant-Kels JM, Dadras SS. 2017. Identification of microRNAs associated with invasive and aggressive phenotype in cutaneous melanoma by next-generation sequencing. Lab Invest 97: 636-648.

Bastian BC, Olshen AB, LeBoit PE, Pinkel D. 2003. Classifying melanocytic tumors based on DNA copy number changes. Am J Pathol 163: 1765-70.

Boiko PE, of the Board PMW, Piepkorn MW. 1994. Reliability of skin biopsy pathology. J Am Board Fam Pract 7: 371-4.

Boyle GM, Woods SL, Bonazzi VF, Stark MS, Hacker E, Aoude LG, Dutton-Regester K, Cook AL, Sturm RA, Hayward NK. 2011. Melanoma cell invasiveness is regulated by miR-211 suppression of the BRN2 transcription factor. Pigment Cell Melanoma Res 24: 525-537.

Brochez L, Verhaeghe E, Grosshans E, Haneke E, Piérard G, Ruiter D, Naeyaert J. 2002. Inter-observer variation in the histopathological diagnosis of clinically suspicious pigmented skin lesions. $J$ Pathol 196: $459-466$.

Buchbinder EI, Flaherty KT. 2016. Biomarkers in Melanoma: Lessons from Translational Medicine. Trends Cancer 2: 305-312.

Chen J, Zhang X, Lentz C, Abi-Daoud M, Paré GC, Yang X, Feilotter HE, Tron VA. 2011. miR-193b Regulates Mcl-1 in Melanoma. Am J Pathol 179: 2162-2168.

Clarke LE, Flake DD, Busam K, Cockerell C, Helm K, McNiff J, Reed J, Tschen J, Kim J, Barnhill R, et al. 2017. An independent validation of a gene expression signature to differentiate malignant melanoma from benign melanocytic nevi. Cancer 123: 617-628.

Conway JR, Lex A, Gehlenborg N. 2017. UpSetR: an R package for the visualization of intersecting sets and their properties. Bioinformatics 33: 2938-2940. 
Corona R, Mele A, Amini M, Rosa DG, Coppola G, Piccardi P, Fucci M, Pasquini P, Faraggiana T. 1996. Interobserver variability on the histopathologic diagnosis of cutaneous melanoma and other pigmented skin lesions. J Clin Oncol Off J Am Soc Clin Oncol 14: 1218-1223.

Ebert MS, Sharp PA. 2012. Roles for microRNAs in conferring robustness to biological processes. Cell 149: $515-524$.

Elder DE, Piepkorn M, Barnhill RL, Longton GM, Nelson HD, Knezevich SR, Pepe MS, Carney PA, Titus LJ, Onega T, et al. 2018. Pathologist Characteristics Associated with Accuracy and Reproducibility of Melanocytic Skin Lesion Interpretation. J Am Acad Dermatol 79: 52-59.e5. Elmore JG, Barnhill RL, Elder DE, Longton GM, Pepe MS, Reisch LM, Carney PA, Titus LJ, Nelson HD, Onega T, et al. 2017. Pathologists' diagnosis of invasive melanoma and melanocytic proliferations: observer accuracy and reproducibility study. BMJ 357: j2813.

Farmer ER, Gonin R, Hanna MP. 1996. Discordance in the histopathologic diagnosis of melanoma and melanocytic nevi between expert pathologists. Hum Pathol 27: 528-531.

Ferrara G, De Vanna AC. 2016. Fluorescence In Situ Hybridization for Melanoma Diagnosis. Am J Dermatopathol 38: 253-269.

Gaudi S, Zarandona JM, Raab SS, English JC, Jukic DM. 2013. Discrepancies in dermatopathology diagnoses: The role of second review policies and dermatopathology fellowship training. J Am Acad Dermatol 68: 119-128.

Gerami P, Mafee M, Lurtsbarapa T, Guitart J, Haghighat Z, Newman M. 2010. Sensitivity of Fluorescence In Situ Hybridization for Melanoma Diagnosis Using RREB1, MYB, Cep6, and 11q13 Probes in Melanoma Subtypes. Arch Dermatol 146: 273-8.

Gershenwald JE, Scolyer RA, Hess KR, Sondak VK, Long G V, Ross MI, Lazar AJ, Faries MB, Kirkwood JM, McArthur GA, et al. 2017. Melanoma staging: Evidence-based changes in the American Joint Committee on Cancer eighth edition cancer staging manual. CA Cancer J Clin 67: $472-492$.

Guyon I, Elisseeff A. 2003. An Introduction to Variable and Feature Selection. J Mach Learn Res 3: 
$1157-1182$.

Hanniford D, Segura MF, Zhong J, Philips E, Jirau-Serrano X, Darvishian F, Berman RS, Shapiro RL, Pavlick AC, Brown B, et al. 2015. Identification of metastasis-suppressive microRNAs in primary melanoma. J Natl Cancer Inst 107.

Hawkes JE, Nguyen GH, Fujita M, Florell SR, Callis Duffin K, Krueger GG, O’Connell RM. 2016. microRNAs in Psoriasis. J Invest Dermatol 136: 365-371.

He Z, Yu W. 2010. Stable feature selection for biomarker discovery. Comput Biol Chem 34: 215-225.

Heenan PJ, Matz LR, Blackwell JB, Kelsall GR, Singh A, ten Seldam RE, Holman CD. 1984. Interobserver variation between pathologists in the classification of cutaneous malignant melanoma in Western Australia. Histopathology 8: 717-729.

Horvath S, Dong J. 2008. Geometric interpretation of gene coexpression network analysis. PLoS Comput Biol 4: e1000117.

Jarry J, Schadendorf D, Greenwood C, Spatz A, van Kempen LC. 2014. The validity of circulating microRNAs in oncology: five years of challenges and contradictions. Mol Oncol 8: 819-829.

Jayawardana K, Schramm S-J, Tembe V, Mueller S, Thompson JF, Scolyer RA, Mann GJ, Yang J. 2016. Identification, Review, and Systematic Cross-Validation of microRNA Prognostic Signatures in Metastatic Melanoma. J Invest Dermatol 136: 245-254.

Jiang L, Lv X, Li J, Li J, Li X, Li W, Li Y. 2012. The status of microRNA-21 expression and its clinical significance in human cutaneous malignant melanoma. Acta Histochem 114: 582-588.

Judson RL, Greve TS, Parchem RJ, Blelloch R. 2013. MicroRNA-based discovery of barriers to dedifferentiation of fibroblasts to pluripotent stem cells. Nat Struct Mol Biol 20: 1227-1235.

Jukic DM, Rao UNM, Kelly L, Skaf JS, Drogowski LM, Kirkwood JM, Panelli MC. 2010. Microrna profiling analysis of differences between the melanoma of young adults and older adults. $J$ Transl Med 8: 27.

Jung M, Schaefer A, Steiner I, Kempkensteffen C, Stephan C, Erbersdobler A, Jung K. 2010. Robust microRNA stability in degraded RNA preparations from human tissue and cell samples. Clin Chem 
56: $998-1006$.

Kappelmann M, Kuphal S, Meister G, Vardimon L, Bosserhoff A-K. 2013. MicroRNA miR-125b controls melanoma progression by direct regulation of c-Jun protein expression. Oncogene 32: 2984-2991.

Komina A, Palkina N, Aksenenko M, Tsyrenzhapova S, Ruksha T. 2016. Antiproliferative and ProApoptotic Effects of MiR-4286 Inhibition in Melanoma Cells. PLoS One 11: e0168229.

Kozubek J, Ma Z, Fleming E, Duggan T, Wu R, Shin D-G, Dadras SS. 2013. In-Depth Characterization of microRNA Transcriptome in Melanoma. PLoS One 8: e72699.

Kursa MB. 2014. Robustness of Random Forest-based gene selection methods. BMC Bioinformatics 15: 8.

Kursa MB, Rudnicki WR. 2010. Feature Selection with the Boruta Package. J Stat Softw 36: 1-13.

Langfelder P, Horvath S. 2008. WGCNA: an R package for weighted correlation network analysis. BMC Bioinformatics 9: 559.

Langmead B, Trapnell C, Pop M, Salzberg SL. 2009. Ultrafast and memory-efficient alignment of short DNA sequences to the human genome. Genome Biol 10: R25.

Latchana N, Del Campo SEM, Grignol VP, Clark JR, Albert SP, Zhang J, Wei L, Aldrink JH, Nicol KK, Ranalli MA, et al. 2017. Classification of Indeterminate Melanocytic Lesions by MicroRNA Profiling. Ann Surg Oncol 24: 347-354.

Latchana N, Ganju A, Howard JH, Carson WE. 2016. MicroRNA dysregulation in melanoma. Surg Oncol 25: 184-189.

Leachman SA, Koon SM, Korcheva VB, White KP. 2017. Assessing Genetic Expression Profiles in Melanoma Diagnosis. Dermatol Clin 35: 537-544.

Lee RC, Feinbaum RL, Ambros V. 1993. The C. elegans heterochronic gene lin-4 encodes small RNAs with antisense complementarity to lin-14. Cell 75: 843-54.

Levy C, Khaled M, Iliopoulos D, Janas MM, Schubert S, Pinner S, Chen P-H, Li S, Fletcher AL, Yokoyama S, et al. 2010. Intronic miR-211 assumes the tumor suppressive function of its host gene 
in melanoma. Mol Cell 40: 841-849.

Li C, Gao Y, Zhang K, Chen J, Han S, Feng B, Wang R, Chen L. 2015. Multiple Roles of MicroRNA100 in Human Cancer and its Therapeutic Potential. Cell Physiol Biochem 37: 2143-2159.

Li H, Durbin R. 2009. Fast and accurate short read alignment with Burrows-Wheeler transform.

Bioinformatics 25: 1754-1760.

Li J, Tran M, Siwabessy J. 2016. Selecting Optimal Random Forest Predictive Models: A Case Study on Predicting the Spatial Distribution of Seabed Hardness. PLoS One 11: e0149089.

Love MI, Huber W, Anders S. 2014. Moderated estimation of fold change and dispersion for RNA-seq data with DESeq2. Genome Biol 15: 550.

Lui JH, Nowakowski TJ, Pollen AA, Javaherian A, Kriegstein AR, Oldham MC. 2014. Radial glia require PDGFD-PDGFR $\beta$ signalling in human but not mouse neocortex. Nature 515: 264-268.

Margue C, Philippidou D, Reinsbach SE, Schmitt M, Behrmann I, Kreis S. 2013. New target genes of MITF-induced microRNA-211 contribute to melanoma cell invasion. PLoS One 8: e73473.

Mazar J, DeYoung K, Khaitan D, Meister E, Almodovar A, Goydos J, Ray A, Perera RJ. 2010. The Regulation of miRNA-211 Expression and Its Role in Melanoma Cell Invasiveness ed. V. Cheriyath. PLoS One 5: e13779.

Minca EC, Al-Rohil RN, Wang M, Harms PW, Ko JS, Collie AM, Kovalyshyn I, Prieto VG, Tetzlaff MT, Billings SD, et al. 2016. Comparison between melanoma gene expression score and fluorescence in situ hybridization for the classification of melanocytic lesions. Mod Pathol 29: 832843.

Mnich K, Rudnicki WR. 2017. All-relevant feature selection using multidimensional filters with exhaustive search. arXiv 1705.05756.

Mumford SL, Towler BP, Pashler AL, Gilleard O, Martin Y, Newbury SF. 2018. Circulating MicroRNA Biomarkers in Melanoma: Tools and Challenges in Personalised Medicine. Biomolecules 8: 21.

Nair VS, Maeda LS, Ioannidis JPA. 2012. Clinical Outcome Prediction by MicroRNAs in Human Cancer: A Systematic Review. JNCI J Natl Cancer Inst 104: 528-540. 
Niebling MG, Haydu LE, Karim RZ, Thompson JF, Scolyer RA. 2014. Pathology Review Significantly Affects Diagnosis and Treatment of Melanoma Patients: An Analysis of 5011 Patients Treated at a Melanoma Treatment Center. Ann Surg Oncol 21: 2245-2251.

Parchem RJ, Ye J, Judson RL, LaRussa MF, Krishnakumar R, Blelloch A, Oldham MC, Blelloch R. 2014. Two miRNA clusters reveal alternative paths in late-stage reprogramming. Cell Stem Cell 14: $617-631$.

Pogribny IP. 2018. MicroRNAs as biomarkers for clinical studies. Exp Biol Med 243: 283-290.

Raya L, Sidi Y, Avni D. 2012. Aberrations in the micro-RNA biogenesis machinery and the emerging roles of micro-RNAs in the pathogenesis of cutaneous malignant melanoma. Pigment Cell Melanoma Res 25: 740-757.

Reddy A, Growney JD, Wilson NS, Emery CM, Johnson JA, Ward R, Monaco KA, Korn J, Monahan JE, Stump MD, et al. 2015. Gene Expression Ratios Lead to Accurate and Translatable Predictors of DR5 Agonism across Multiple Tumor Lineages. PLoS One 10: e0138486.

Reddy KB. 2015. MicroRNA (miRNA) in cancer. Cancer Cell Int 15: 38.

Ritchie ME, Phipson B, Wu D, Hu Y, Law CW, Shi W, Smyth GK. 2015. limma powers differential expression analyses for RNA-sequencing and microarray studies. Nucleic Acids Res 43: e47.

Rodríguez-Cerdeira C, Molares-Vila A, Carnero-Gregorio M, Corbalán-Rivas A. Recent advances in melanoma research via “omics” platforms. J Proteomics 10.1016/j.jprot.2017.11.005.

Saeys Y, Inza I, Larrañaga P. 2007. A review of feature selection techniques in bioinformatics. Bioinformatics 23: 2507-2517.

Sand M, Skrygan M, Sand D, Georgas D, Gambichler T, Hahn SA, Altmeyer P, Bechara FG. 2013. Comparative microarray analysis of microRNA expression profiles in primary cutaneous malignant melanoma, cutaneous malignant melanoma metastases, and benign melanocytic nevi. Cell Tissue Res 351: 85-98.

Satzger I, Mattern A, Kuettler U, Weinspach D, Niebuhr M, Kapp A, Gutzmer R. 2012. microRNA-21 is upregulated in malignant melanoma and influences apoptosis of melanocytic cells. Exp Dermatol 
21: $509-514$.

Shain AH, Joseph NM, Yu R, Benhamida J, Liu S, Prow T, Ruben B, North J, Pincus L, Yeh I, et al. 2018. Genomic and Transcriptomic Analysis Reveals Incremental Disruption of Key Signaling Pathways during Melanoma Evolution. Cancer Cell 34: 45-55.

Shain AH, Yeh I, Kovalyshyn I, Sriharan A, Talevich E, Gagnon A, Dummer R, North J, Pincus L, Ruben B, et al. 2015. The Genetic Evolution of Melanoma from Precursor Lesions. $N$ Engl J Med 373: $1926-1936$.

Sheinerman KS, Umansky SR. 2013. Circulating cell-free microRNA as biomarkers for screening, diagnosis and monitoring of neurodegenerative diseases and other neurologic pathologies. Front Cell Neurosci 7: 150.

Shoo AB, Sagebiel RW, Mohammed K-S. 2010. Discordance in the histopathologic diagnosis of melanoma at a melanoma referral center. J Am Acad Dermatol 62: 751-756.

Talevich E, Shain AH, Botton T, Bastian BC. 2016. CNVkit: Genome-Wide Copy Number Detection and Visualization from Targeted DNA Sequencing. PLoS Comput Biol 12: e1004873.

Valastyan S, Weinberg RA. 2010. miR-31: A crucial overseer of tumor metastasis and other emerging roles. Cell Cycle 9: 2124-2129.

Wallace E, Lowry J, Smith SM, Fahey T. 2013. The epidemiology of malpractice claims in primary care: a systematic review. BMJ Open 3: e002929.

Wandler A, Riber-Hansen R, Hager H, Hamilton-Dutoit SJ, Schmidt H, Nielsen BS, Stougaard M, Steiniche T. 2017. Quantification of microRNA-21 and microRNA-125b in melanoma tissue. Melanoma Res 27: 417-428.

Wang L, Rao M, Fang Y, Hameed M, Viale A, Busam K, Jhanwar SC. 2013. A genome-wide highresolution array-cgh analysis of cutaneous melanoma and comparison of array-cgh to fish in diagnostic evaluation. J Mol Diagnostics 15: 581-591.

Wenric S, Shemirani R. 2018. Using Supervised Learning Methods for Gene Selection in RNA-Seq CaseControl Studies. Front Genet 9: DOI: 10.3389/fgene.2018.00297. 
Witwer KW, Halushka MK. 2016. Towards the Promise of microRNAs - Enhancing reproducibility and rigor in microRNA research. RNA Biol 13: 1103-1116.

Xu Y, Brenn T, Brown ERS, Doherty V, Melton DW. 2012. Differential expression of microRNAs during melanoma progression: miR-200c, miR-205 and miR-211 are downregulated in melanoma and act as tumour suppressors. Br J Cancer 106: 553-561.

Zhang J, Lu L, Xiong Y, Qin W, Zhang Y, Qian Y, Jiang H, Liu W. 2014. MLK3 promotes melanoma proliferation and invasion and is a target of microRNA-125b. Clin Exp Dermatol 39: 376-384.

Key Statistics for Melanoma Skin Cancer. https://www.cancer.org/cancer/melanoma-skincancer/about/key-statistics.html (Accessed December 19, 2018). 